\title{
The modeling and simulation of collision protection system between the driver of non-motor vehicle and car door
}

\author{
Yujun Lu*, Jiatao Lai and Yan Feng
}

\begin{abstract}
Collision between the driver of non-motor vehicle and car door often happens when the car driver or the passenger in the parking car opens the door suddenly ignoring a non-motor vehicle passing by. The collision protection system is proposed by installing an airbag in the car door. When the collision accident between the driver of non-motor vehicle and the car is unavoidable, the airbag will automatically deploy to provide head protection for the driver of non-motor vehicle. Utilizing HyperWorks and LS-DYNA, the finite element model of the collision is established to simulate the situations of installing airbag or not with the door on different opening angles $\left(60^{\circ}, 70^{\circ}\right)$ and to verify the feasibility of the proposed method.
\end{abstract}

Keywords: Rider-car door collision, Protection system, Airbag, Different opening angles, The finite element model

\section{Introduction}

The pedestrian-car crash is one of main types of traffic accidents, and the rate of casualties is high for this kind of accident in China [1,2]. The pedestrian safety protection has been studied by researchers in many countries and is also the focus of passive safety protection research [3-5]. Thus, it is urgent and of great practical significance to carry out the research on pedestrian safety protection $[6,7]$.

Most of related research is focused on pedestrian safety protection of frontal crash between pedestrian and vehicle $[8,9]$. However, since the Chinese invented the electric bicycles in 1996 which are categorized as non-motor vehicles and usually run at a speed of $35 \mathrm{~km} / \mathrm{h}$ or even higher, China has been the largest producer and consumer of the electric bikes. Owing to high speed of the electric bicycles, there are often some hidden accidents, such as "door of the car opened resulting to death" (as shown in Fig. 1). The collision accident between the driver of non-motor vehicle and car door will occur when the driver or the passenger in the parking car opens the door suddenly ignoring a bicycle or an electric bicycle passing by. For simplicity, in the following paper, a rider is used to represent the driver of a non-motor vehicle. If the rider collides with the door at a very high speed, the consequence will be very serious and may lead to serious injury, even to death. Just some research of the collision warning system has been carried out by some researchers $[10,11]$, while the rider-car door collision protection system has not been studied yet.

A rider-car door collision protection system is proposed in this paper by installing an airbag in a car door. When the collision is unavoidable, the airbag will automatically deploy to provide head protection for the rider. The finite element model of rider-car door collision utilizing HyperWorks and LS-DYNA is established to simulate the collision situations with airbag or not in the door on different opening angles $\left(60^{\circ}, 70^{\circ}\right)$. The simulation results verify the feasibility of the proposed method.

* Correspondence: Iuet_lyj@163.com

Faculty of Mechanical Engineering and Automation, Zhejiang Sci-Tech

University, Hangzhou, China 


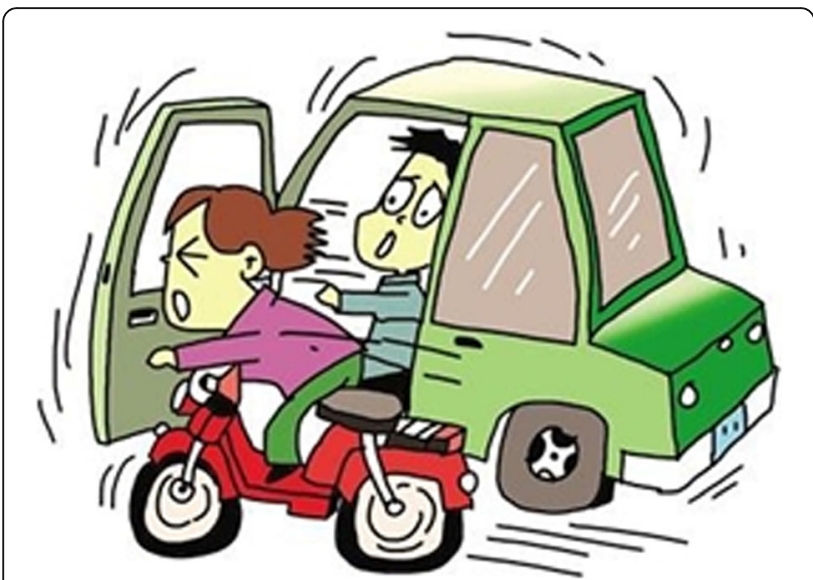

Fig. 1 Diagram of the collision

The rest of this paper is organized as follows. In Section 2, the design of the rider-car door collision protection system is put forward. Section 3 gives the finite element modeling process of the protection system. Section 4 presents the simulation results of rider-car door collision without the protection system. Section 5 presents the simulation results of rider-car door collision with the protection system. The paper is concluded in Section 6.

\section{Design of the rider-car door collision protection system}

The rider maintains a horizontal distance with the body of a car in the process of driving. The position that the rider collided with the opening door is usually the left door of the parking car. The front wheel of the nonmotor vehicle contacts with the door first, and then the rider begins to move forward and collides with the door because of inertia.

The rider-car door collision protection system proposed in the paper consists of infrared sensor, distance sensor, angle controller, displacement sensor, controller,

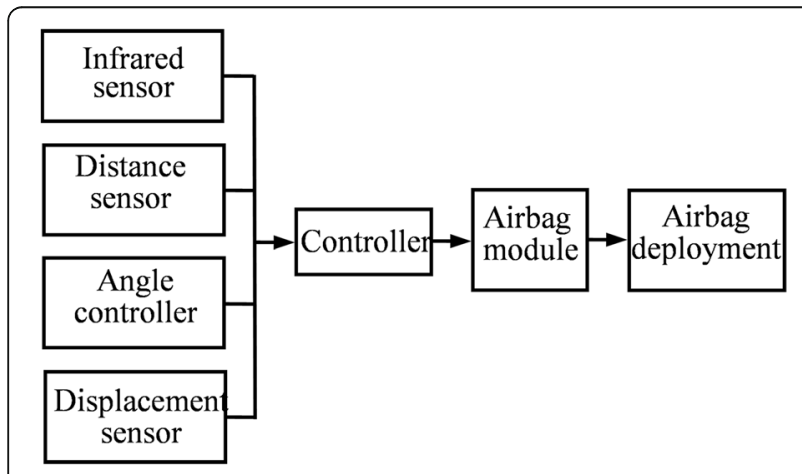

Fig. 2 The rider-car door collision protection system airbag, and airbag module. The system is presented in Fig. 2.

In the rider-car door collision protection system, the infrared sensor is used to detect whether someone is behind the door. The distance sensor is used to detect the relative distance between the opening door and the rider. According to the opening angle of the door, the controller uses the angle controller to keep the direction of the distance sensor always backward and keep parallel to the body of the car. The controller can calculate the speed of the moving object by using the distance sensor to detect the distance value of the moving object at different moments and compare it with the critical value of distance and speed which have been already set in advance. At the same time, the controller can calculate the opening angle of the door in real time by using the displacement sensor to detect the displacement value of door opening at different moments and compare it with the critical value of the door opening angle which has been already set in advance. When the relative distance between the opening door and the non-motor vehicle, the speed of the non-motor vehicle, and the door opening angle all reach the critical value, it is determined that the airbag needs to deploy immediately to achieve the protection function.

The purpose of the system is to provide head protection for the rider. The control device of the induction system is installed in the lower side of the inner window of the door. The coverage area of the fully deployed airbag is mainly concentrated on the upper part

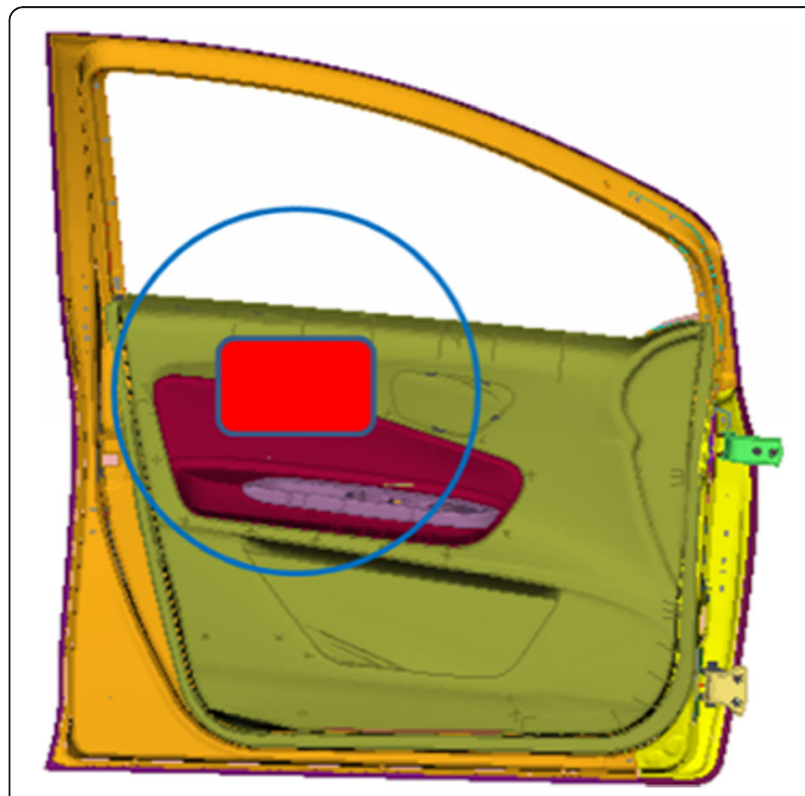

Fig. 3 The position of the airbag assembly 


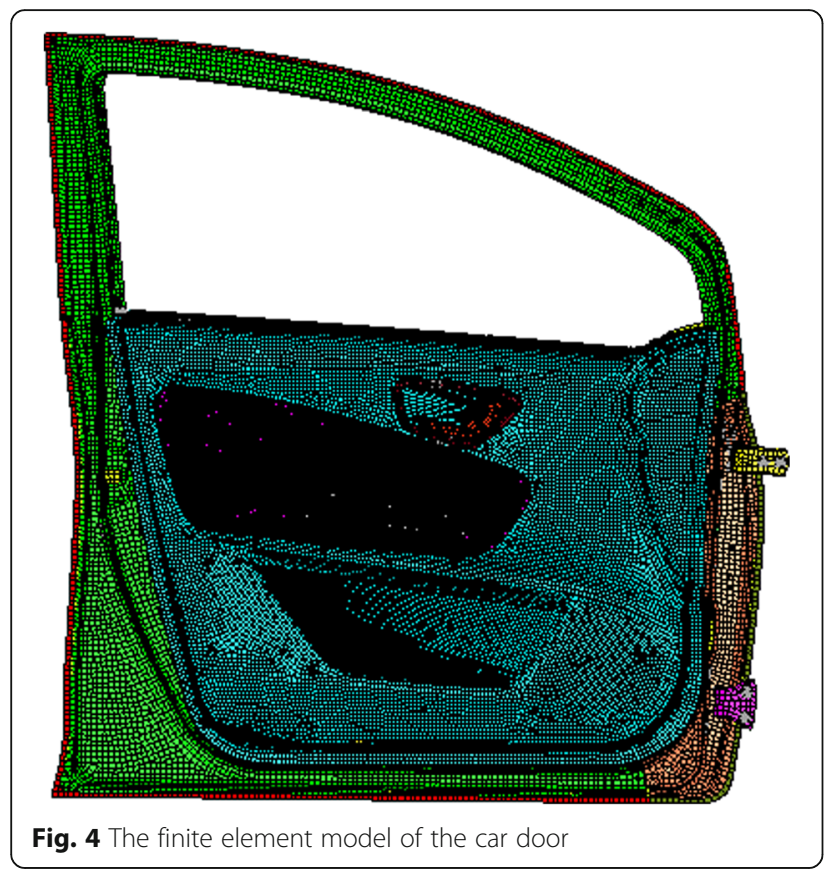

of the inner door. Considering the large coverage area of the airbag, a circular airbag structure is adopted. This kind of airbag is easy to arrange and has a large coverage area, which is suitable for head protection. According to the interior structure of the door, the position of the airbag assembly is set in the red box and the coverage area after the expansion is shown in the blue area in Fig. 3.

\section{Finite element modeling of the rider-car door collision protection system}

\subsection{The finite element model of the car door}

The door of an SUV is chosen as the research object for simulation analysis. The finite element model of the door is established. The outer panel, the inner plate, and other accessories of the door are set up by shell element. Mesh quality is especially important for the establishment of finite element, which directly affects the accuracy of the calculation result [12]. In the paper, the average size of a door assembly grid is $10 \mathrm{~mm}$. The corresponding materials and attributes are given to the model according to the material list of parts of the door. The material of the door hinge is SAPH440, the material of the inner panel is DC03, the material of the outer panel is $\mathrm{B} 180 \mathrm{H} 1$, and the materials of the interior trimming panel are $\mathrm{PC}+$ ABS-GF20 and PBT + ASA-GF30. For the door assembly process, hemming connection is set between the inner panel and the outer panel, the solder joint connection is set between the inner plate and the reinforcing plate, the buckle connection is set between the interior trimming panel and its accessories. The finite element model of the door is shown in Fig. 4.

Due to the simplification of parts in the process of modeling, the finite element model of the door will be greatly different from the actual door in mass after giving materials to the parts. The location of the center of mass will also have large deviation, so the door should be counterweighted [13]. As shown in Fig. 5, the door assembly is counterweighted with mass units until the weight and the center of mass are equal to the actual state. Six degrees of freedom of door hinge and the installation point of car body are constrained to simulate the relationship between the door and the body of car when the vehicle stops. The rotating hinge is set at the two hinge connections of the door to achieve their rotation relationship.

\subsection{The finite element model of a standard rider's head}

The head model is simulated due to the greatest damage to the head of the rider in the rider-car door collision. According to GB/T 24550-2009, for "the protection of motor vehicle for pedestrians in the event of a collision",

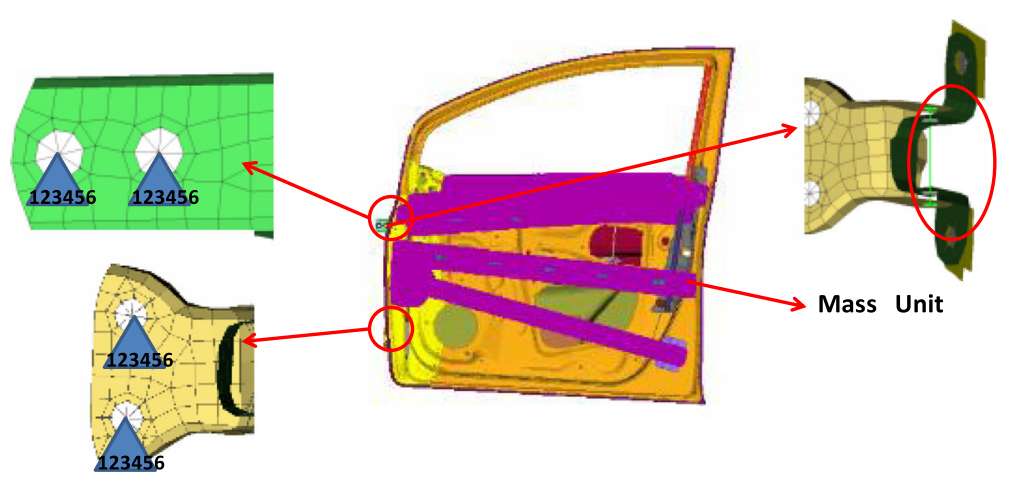

Fig. 5 Counterweight of the car door assembly with mass units 


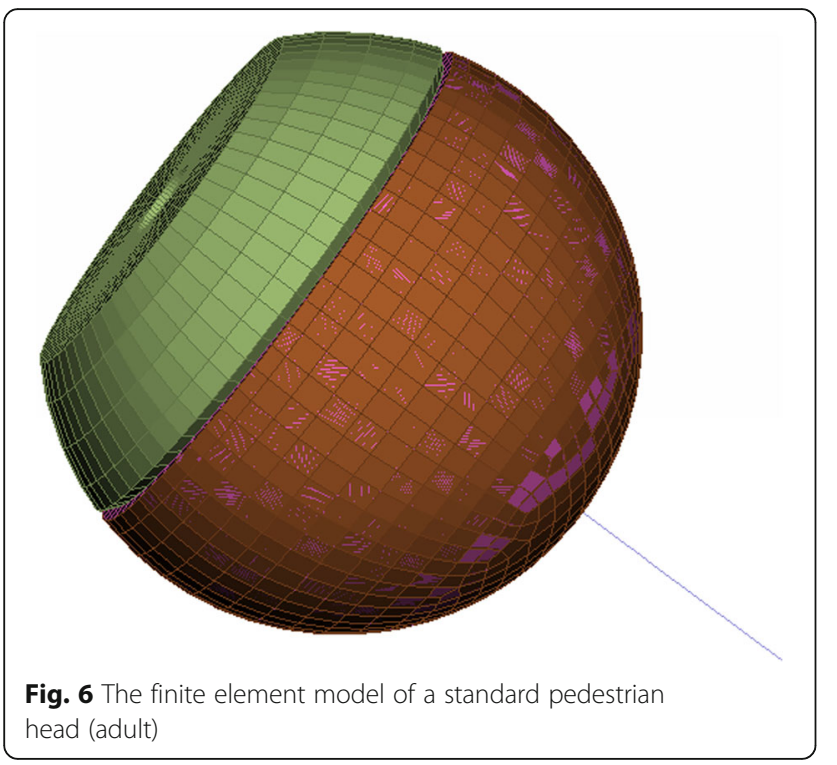

the synthetic acceleration of dummy head should make the value of head injury criteria (HIC) below 1000 .

$$
\mathrm{HIC}=\left(t_{2}-t_{1}\right)\left[\frac{1}{\left(t_{2}-t_{1}\right)} \int_{t_{1}}^{t_{2}} a_{r} d t\right]^{2.5}
$$

where $a_{r}$ represents the synthetic acceleration at the center of dummy head and $t_{1}$ and $t_{2}$ represent the any two moments chosen in the collision process. The value of $a_{r}$ is the multiple of gravitational acceleration, the time interval between $t_{1}$ and $t_{2}$ is less than $36 \mathrm{~ms}$, and $t_{1}$ is less than $t_{2}$.
The finite element model of standard pedestrian head (adult) is shown in Fig. 6. The acceleration sensor unit is set up in the model, which can output head acceleration information. The structure design is carried out to optimize the value of $\mathrm{HIC}$ in the paper.

\subsection{The finite element model of the airbag and box}

The diameter of the airbag in the paper is $606 \mathrm{~mm}$, and the area of the exhaust hole is $250 \mathrm{~mm}^{2}$. The box of the airbag assembly is the mounting bracket of airbag assembly and is installed in the reinforcing plate of the door. The size of the box is $250 \times 165 \times 66$, and the material is $\mathrm{B} 180 \mathrm{H} 1$.

The primer software is used to fold the airbag. The folding way is as follows: the base portion is first folded, the top portion is folded later, and it is rotationally folded finally. The folding process of the airbag is presented in Fig. 7.

The airbag and the box are meshed by HyperMesh. The grid unit of the airbag is triangular with average size of $8 \mathrm{~mm}$. The grid unit of the box is rectangular with average size of $10 \mathrm{~mm}$. The airbag is placed in the box. Rigid connection is used between the inflatable port of the airbag and the bottom of the box. CONTACT AUTOMATIC_SURFACE_TO_SURFACE [14] is used to define the direct contact between the box and the airbag. The finite element model of the airbag and box is shown in Fig. 8.

AIRBAG_WANG_NEFSKE_JETTING is selected as the inflatable model of the airbag, which is of high precision and has a wide range of application. Key cards of the inflatable model in LS-DYNA are shown in Fig. 9.
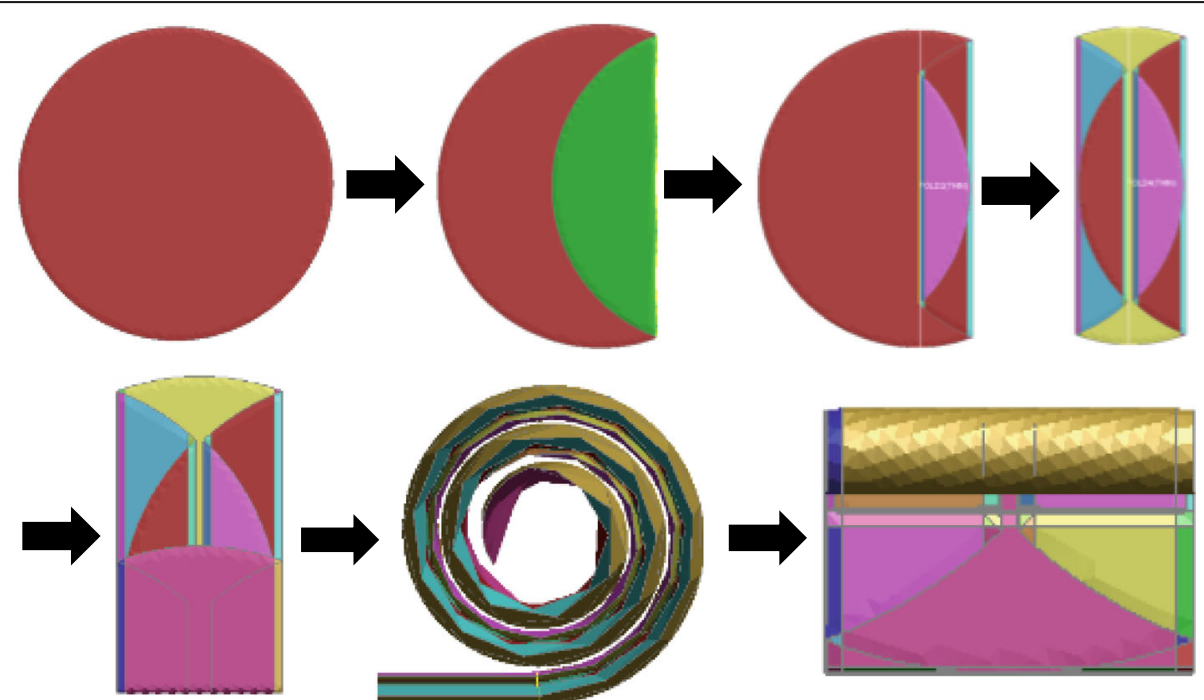

Fig. 7 The folding process of the airbag 


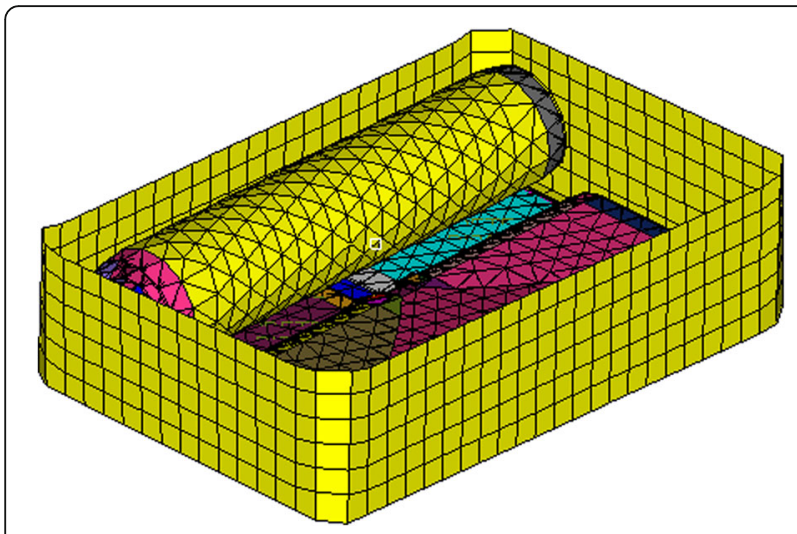

Fig. 8 The finite element model of the airbag and box

Key words mainly define external environment parameters, temperature of gas, size of vent hole, and mass flow rate curve (as shown in Fig. 10), which can better reflect the test performance of the airbag [15]. The airbag is easily penetrated during the contact with a rigid material. CONTACT_AIRBAG_SINGLE_SURFACE is set as the type of self-contact of the airbag [16].

The airbag is fully expanded at about $25 \mathrm{~ms}$, and the thickness of expansion is $246 \mathrm{~mm}$. The fully deployed airbag is shown in Fig. 11.

\section{Analysis of rider-car door collision results}

Simulation analysis of rider-car door collision in the paper is carried out to simulate the accidents that the rider hit the door at $40 \mathrm{~km} / \mathrm{h}$ with the door on different opening angles $\left(60^{\circ}, 70^{\circ}\right)$. The processing of the model is as follows: six degrees of freedom of hinge mounting point are constrained; the rotational degree of freedom between two parts of door hinges is released, the initial speed of adult head dummy is $40 \mathrm{~km} / \mathrm{h}$, surface-tosurface contact is set between the head dummy and the interior trimming panel. After completing the preprocessing of the finite element model, the finite element model of rider-car door collision is exported as a $\mathrm{K}$ file, which is submitted to LS-DYNA solver for calculation. Through the post processing software HyperGraph, the curve and the data are processed, and the result of damage value is obtained.

\subsection{Rider-car door collision on the opening angle of $60^{\circ}$} The door opening angle is $60^{\circ}$, and the rider-car door collision process is shown in Fig. 12. In the case of without airbag protection, the result of head injury value on the opening angle of $60^{\circ}$ is shown in Fig. 13 . The peak acceleration of the adult head dummy is over $250 \mathrm{~g}$ and the value of HIC is 2192, which substantially exceeds the standard value and the rider's head may be injured seriously.

\subsection{Rider-car door collision on the opening angle of $70^{\circ}$}

The door is located at the maximum opening angle of $70^{\circ}$. Six degrees of freedom of a location unit node of a door limiter are constrained. The rider-car door collision process is shown in Fig. 14.

In the case of without airbag protection, the result of head injury value on the opening angle of $70^{\circ}$ is shown in Fig. 15. The peak acceleration of the adult head dummy is over $350 \mathrm{~g}$ and the value of HIC is 3317, which substantially exceeds the standard value and the rider's head may be injured seriously.

According to GB/T 24550-2009, if the value of HIC exceeds 1000 in the collision, it is regarded as serious injury and may lead to danger to the life of the rider. It is shown from the result of the simulation that the value of $\mathrm{HIC}$ is much higher than 1000 and there is danger to the life of the rider without airbag protection in the collision.

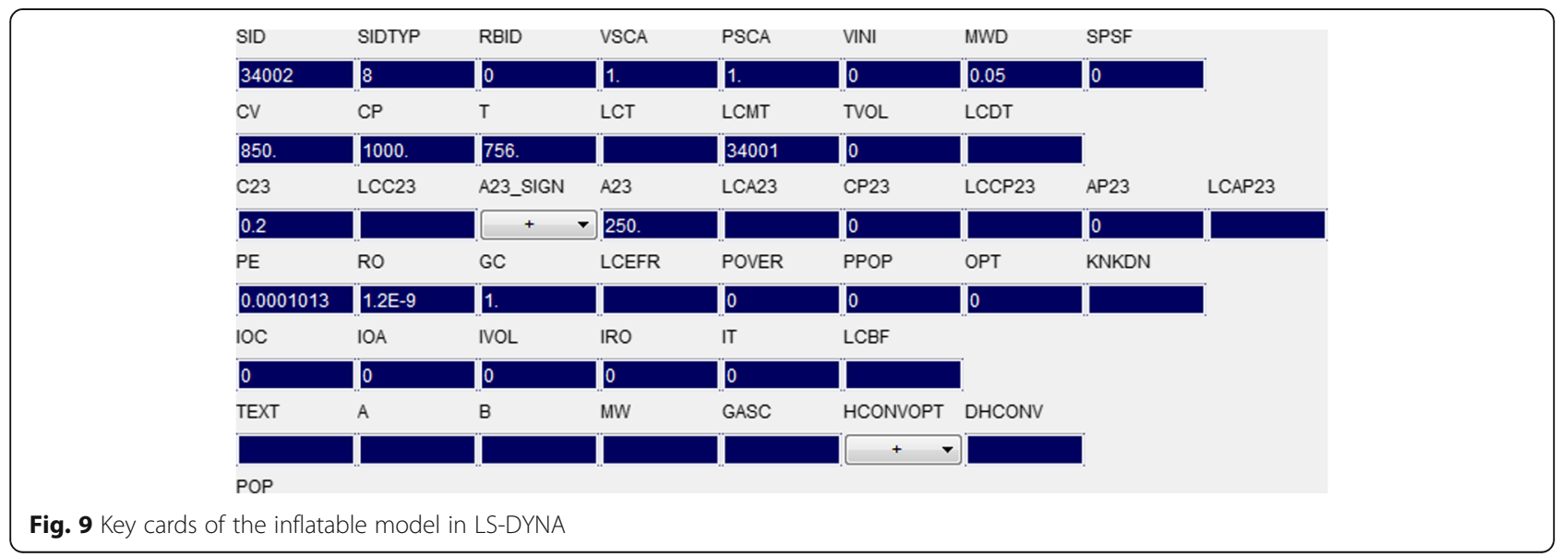




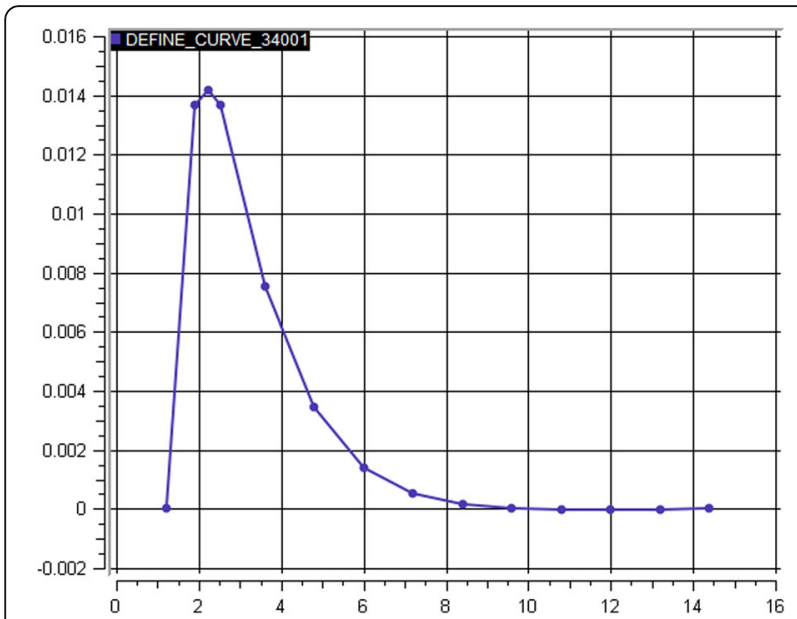

Fig. 10 Mass flow rate curve

\section{Analysis of rider-airbag-car door collision results} It was simulated that a rider collides with the opening door in the condition of no airbag protection. The injury value was very high, which could cause serious injury to the rider's head in actual accidents.

In the paper, simulation analysis of rider-airbag-car door collision is carried out to simulate the accidents that the rider moves at $40 \mathrm{~km} / \mathrm{h}$ towards the car door, and the airbag expands to provide protection with the door on different opening angles $\left(60^{\circ}, 70^{\circ}\right)$. The processing of the model is as follows: six degrees of freedom of the hinge mounting

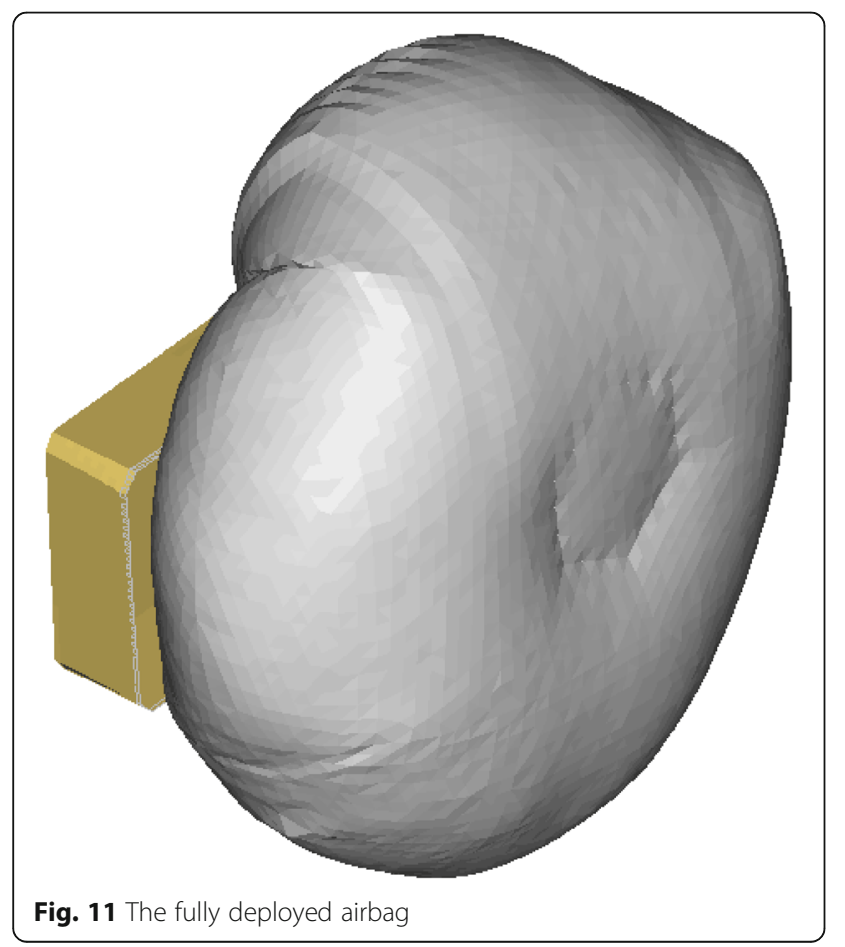

point are constrained, the rotational degree of freedom between two parts of the door hinges is released, the initial speed of the adult head dummy is $40 \mathrm{~km} / \mathrm{h}$, and surfaceto-surface contact is set between the head dummy and the airbag. After completing the pre-processing of the finite element model, the finite element model of rider-airbagcar door collision is exported as a $\mathrm{K}$ file, which is submitted to LS-DYNA solver for calculation. Through the post processing software HyperGraph, the curve and the data are processed, and the result of the damage value is obtained.

\subsection{Rider-airbag-car door collision on the opening angle of $60^{\circ}$}

The door opening angle is $60^{\circ}$, and the rider-airbag-car door collision process is shown in Fig. 16. In the case of with airbag protection, the result of head injury value on the opening angle of $60^{\circ}$ is shown in Fig. 17. The peak acceleration of the adult head dummy is $65 \mathrm{~g}$ and the value of $\mathrm{HIC}$ is 537 .

Compared with that of the rider-car door collision without airbag protection at an opening angle of $60^{\circ}$, the peak acceleration of the adult head dummy decreases from 260 to $65 \mathrm{~g}$ and the value of HIC decreases from 2192 to 537 . It shows that the safety performance of the door with airbag in this collision can effectively meet the requirements for head protection of the rider.

\subsection{Rider-airbag-car door collision on the opening angle of $70^{\circ}$}

The door is located at the maximum opening angle of $70^{\circ}$. Six degrees of freedom of the location unit node of door limiter are constrained. The door-airbag-rider collision process is shown in Fig. 18. In the case of with airbag protection, the result of head injury value on the opening angle of $70^{\circ}$ is shown in Fig. 19. The peak acceleration of the adult head dummy is $80 \mathrm{~g}$ and the value of $\mathrm{HIC}$ is 769 .

Compared with that of the rider-car door collision without airbag protection at an opening angle of $70^{\circ}$, the peak acceleration of the adult head dummy decreases from 375 to $80 \mathrm{~g}$ and the value of HIC decreases from 3317 to 769 . It can be concluded that the safety performance of the door with airbag in this collision can effectively meet the requirements for head protection of the rider.

\section{Conclusions}

A rider-car door collision protection system is proposed in the paper. The finite element model of rider-car door collision is established to simulate the situations of installing airbag or not with the door at different opening angles $\left(60^{\circ}, 70^{\circ}\right)$ and to verify the feasibility of the proposed method. 

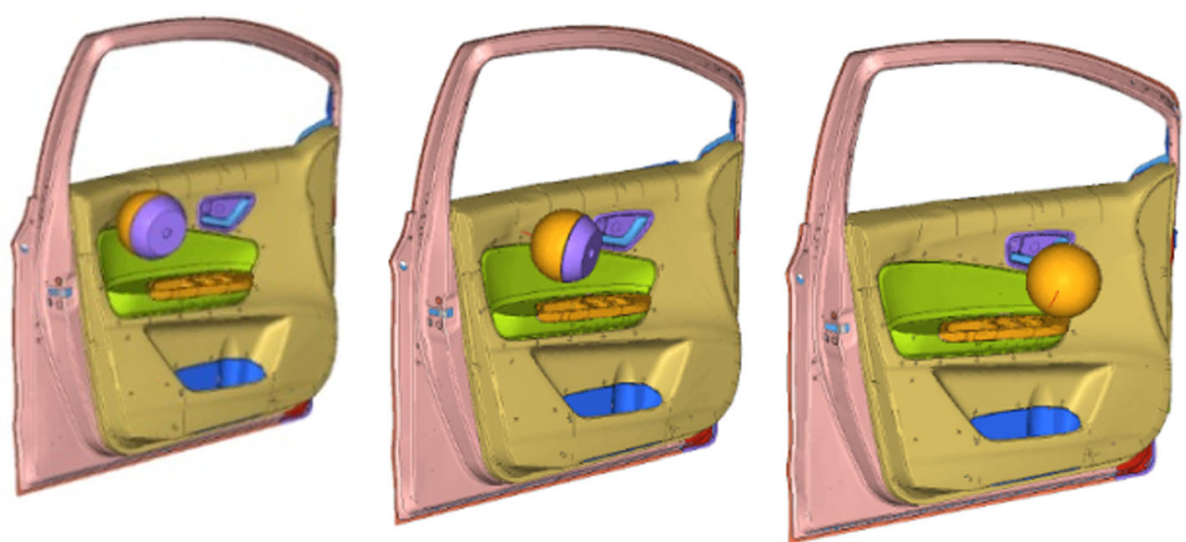

Fig. 12 The rider-car door collision process without the airbag protection at the opening angle of $60^{\circ}$

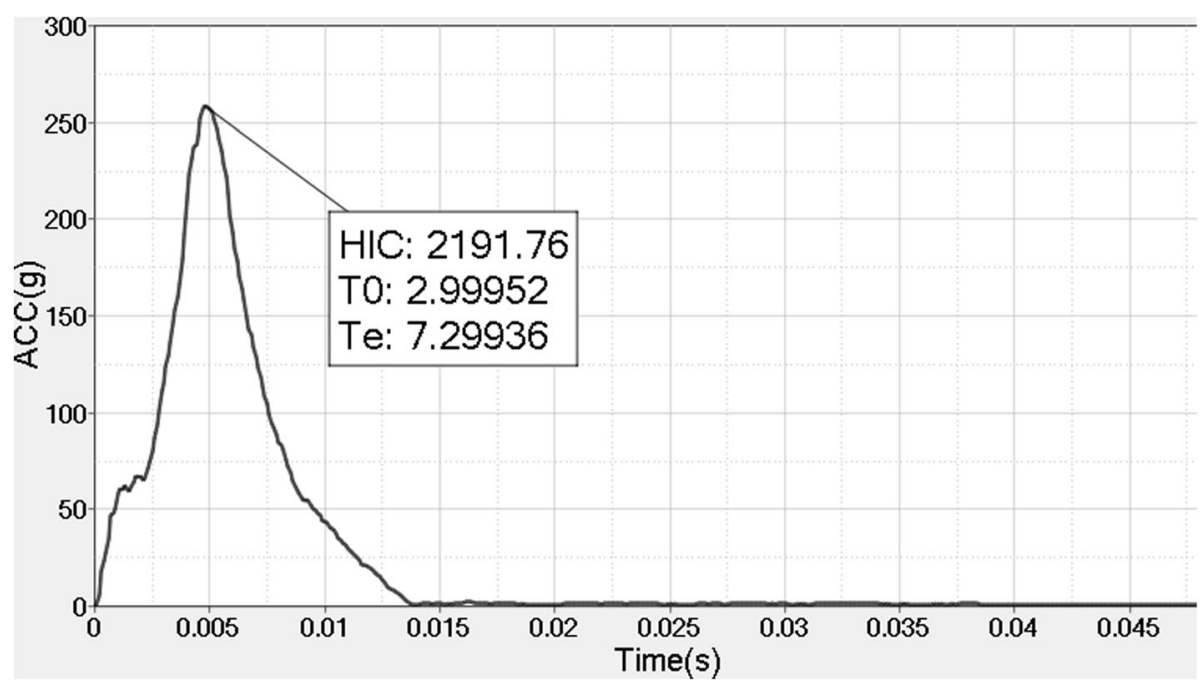

Fig. 13 The result of head injury value without the airbag protection at the opening angle of $60^{\circ}$
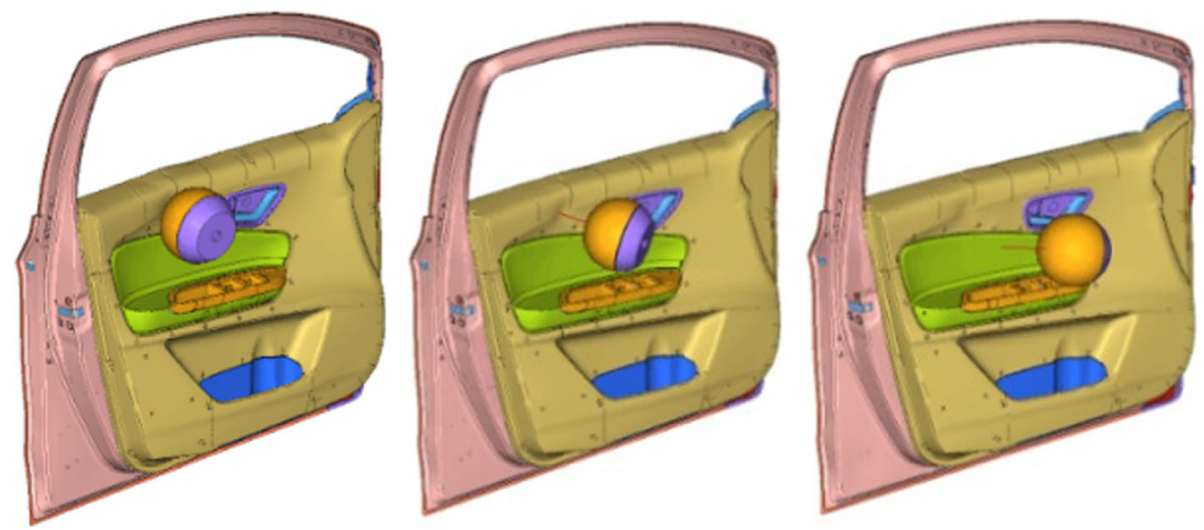

Fig. 14 The rider-car door collision process without the airbag protection at the opening angle of $70^{\circ}$ 


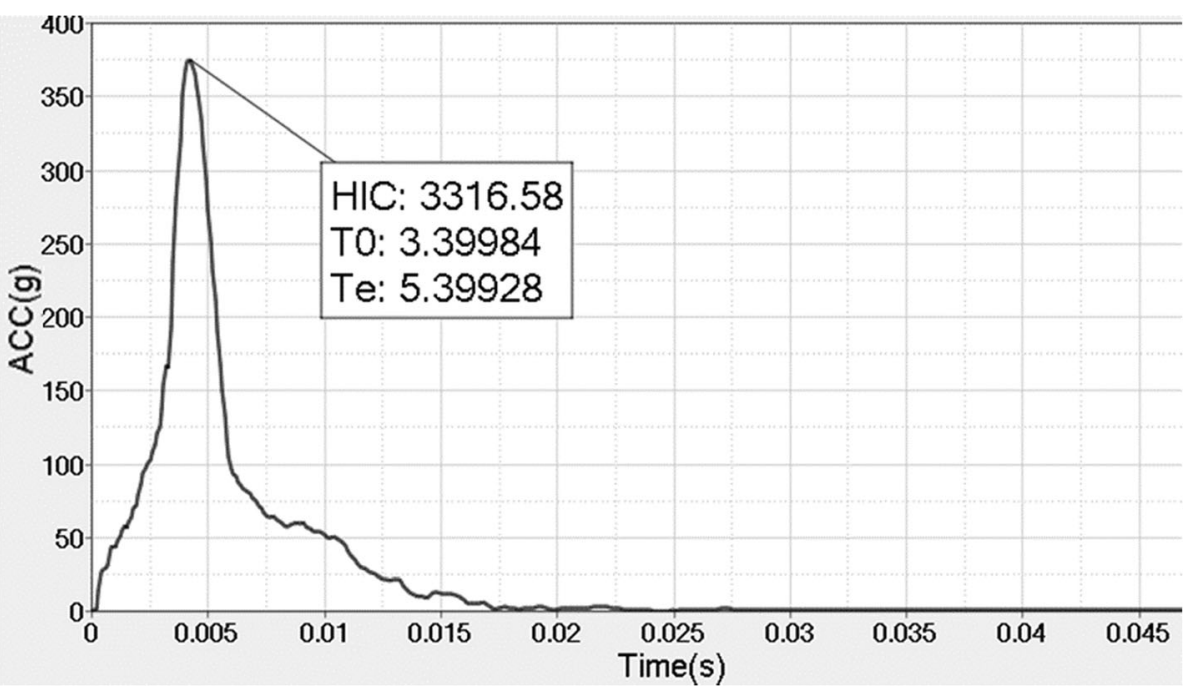

Fig. 15 The result of head injury value without the airbag protection at the opening angle of $70^{\circ}$
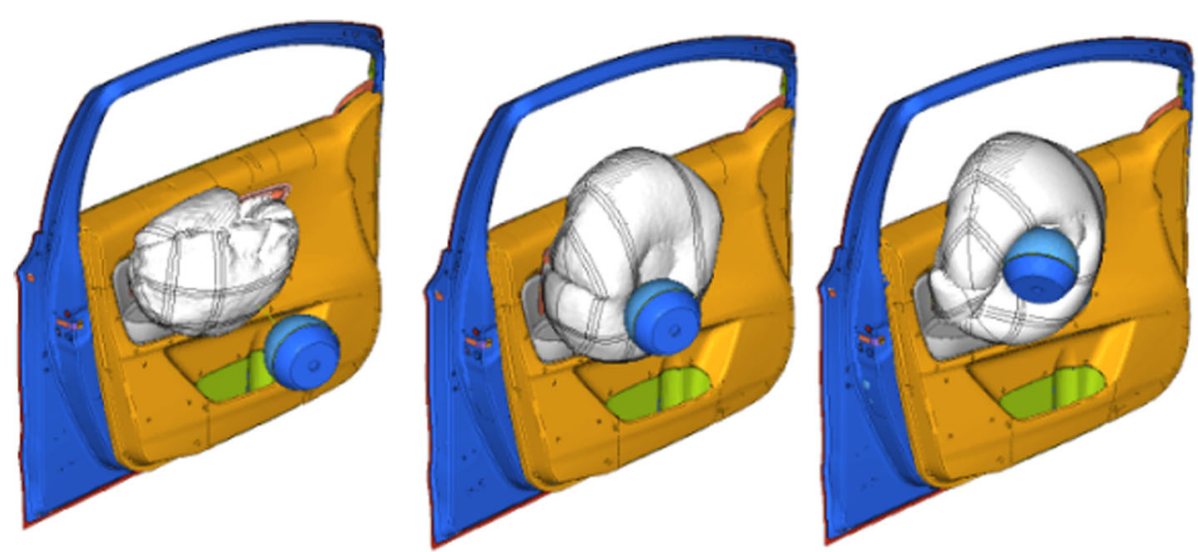

Fig. 16 The rider-airbag-car door collision process with the airbag protection at the opening angle of $60^{\circ}$

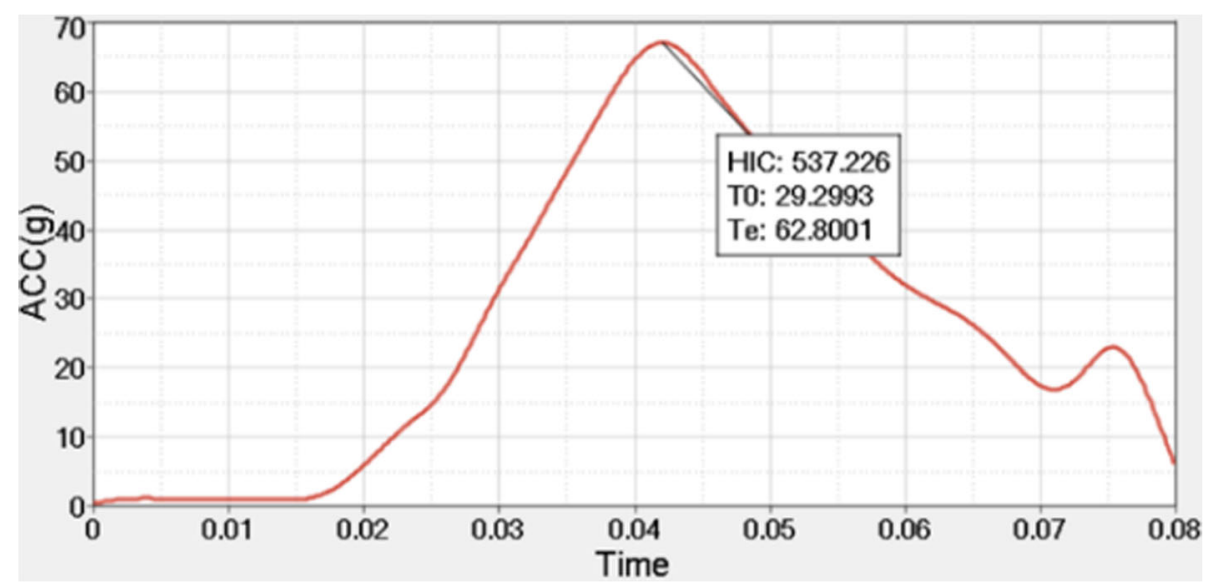

Fig. 17 The result of a head injury value with the airbag protection at the opening angle of $60^{\circ}$ 


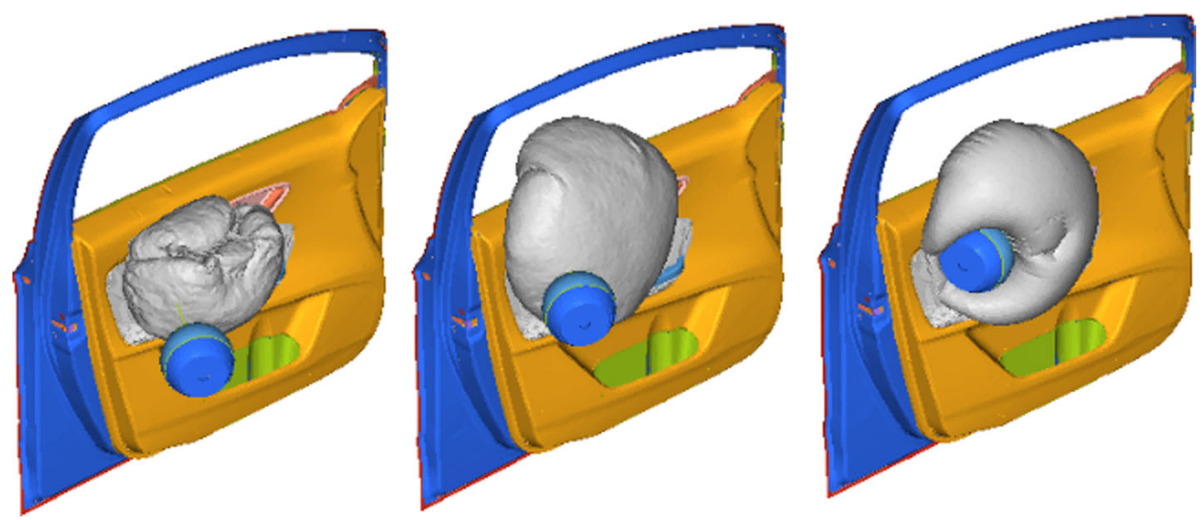

Fig. 18 The rider-airbag-car door collision process with the airbag protection at the opening angle of $70^{\circ}$

The results are as follows:

(1) The head injury value in the rider-car door collision without airbag protection on the opening angles of $60^{\circ}$ and $70^{\circ}$ are 2192 and 3317 , which are much higher than the threshold value of HIC and may lead to life danger of the rider.

(2) The head injury value in the rider-airbag-car door collision with airbag protection on the door opening angles of $60^{\circ}$ and $70^{\circ}$ are 537 and 769 , which are lower than the threshold value of HIC.

(3) The head protection for the rider is greatly enhanced with the door equipped with an airbag. The safety performance of the door with an airbag can effectively meet the requirements for rider's head protection and the values of HIC on different opening angles are in the safe range, which proves the feasibility of the proposed method.

\section{Method}

In this work, a rider-car door collision protection system was studied to protect the rider from serious injury. The rider-car door collision protection system consists of sensors, controllers, and an airbag module installed in car door. To ensure a larger scope of protection as possible, a circular airbag structure was adopted here to buffer percussive force to the rider. HyperWorks and LS-DYNA were utilized to establish the finite element model of the collision and to simulate the situations of installing the airbag or not with the door on $60^{\circ}$ and $70^{\circ}$ opening angles. The simulation results show that the protection system proposed in this paper is effective and the values of HIC to the rider are reduced greatly to the safe range.

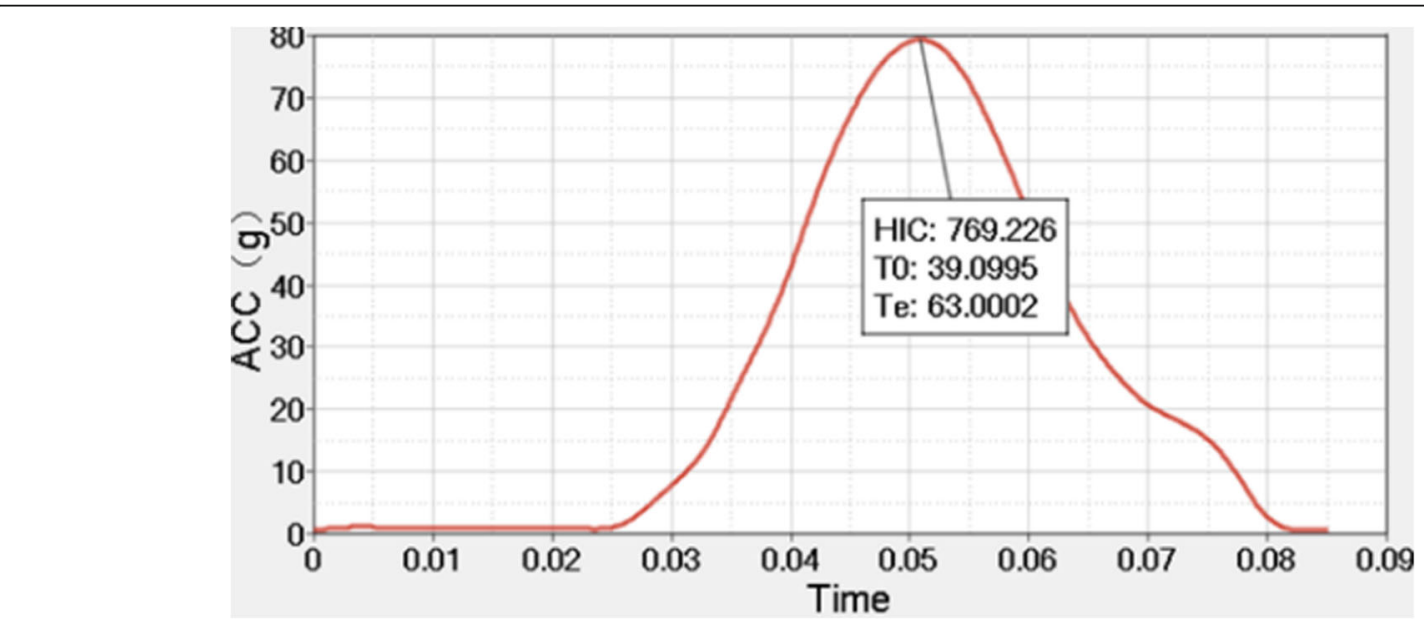

Fig. 19 The result of head injury value with the airbag protection at the opening angle of $70^{\circ}$ 


\section{Acknowledgements}

The research presented in this paper was supported by Ministry of Science and Technology, China, Science Technology Department of Zhejiang Province, and Zhejiang Sci-Tech University.

\section{Funding}

The authors acknowledge the innovation method project of the Ministry of Science and Technology of China (2016IM020100), the Key R \& D project of Zhejiang Province (No. 2018C01074), the commonweal project of Zhejiang Province (No. 2016C31073), and 521 talent project of ZSTU.

\section{Authors' contributions}

$Y L$ proposed the main idea and revised the whole paper. $J L$ designed the rider-car door collision protection system, and YF presented the finite element model of door-pedestrian and analyzed the result. All authors read and approved the final manuscript.

\section{Competing interests}

The authors declare that they have no competing interests.

\section{Publisher's Note}

Springer Nature remains neutral with regard to jurisdictional claims in published maps and institutional affiliations.

Received: 9 March 2018 Accepted: 23 April 2018

Published online: 02 May 2018

\section{References}

1. Q Chen, M Lin, B Dai, et al., Typical pedestrian accident scenarios in China and crash severity mitigation by autonomous emergency braking systems []]. SAE Technical Paper 2015-01-1464 (2015). https://doi.org/10.4271/2015-01-1464

2. J Nie, J Yang, A study of bicyclist kinematics and injuries based on reconstruction of passenger car-bicycle accident in China [J]. Accid. Anal. Prev. 71(2), 50-59 (2014)

3. A Kopczyński, M Ptak, P Harnatkiewicz, The influence of frontal protection system design on pedestrian passive safety [J]. Arch. Civ. Mech. Eng. 11(2), 345-364 (2011)

4. B Depreitere, C Van Lierde, S Maene, et al., Bicycle-related head injury: a study of 86 cases [J]. Accid. Anal. Prev. 36(4), 561-567 (2004)

5. L Raslavičius, L Bazaras, R Keršys, Accident reconstruction and assessment of cyclist's injuries sustained in car-to-bicycle collision [J]. Procedia Eng. 187, 562-569 (2017)

6. E Rosén, $\mathrm{H}$ Stigson, $U$ Sander, Literature review of pedestrian fatality risk as a function of car impact speed [J]. Accid. Anal. Prev. 43, 25-33 (2011)

7. Y Peng, Y Chen, J Yang, et al., A study of pedestrian and bicyclist exposure to head injury in passenger car collisions based on accident data and simulations [J]. Saf. Sci. 50(9), 1749-1759 (2012)

8. G Li, J Yang, C Simms, Safer passenger car front shapes for pedestrians: A computational approach to reduce overall pedestrian injury risk in realistic impact scenarios [J]. Accid. Anal. Prev. 100, 97-110 (2017)

9. G Li, M Lyons, B Wang, et al., The influence of passenger car front shape on pedestrian injury risk observed from German in-depth accident data [J]. Accid. Anal. Prev. 101, 11-21 (2017)

10. P Wang, CY Chan, Vehicle collision prediction at intersections based on comparison of minimal distance between vehicles and dynamic thresholds [J]. IET Intell. Transp. Syst. 11(10), 676-684 (2016)

11. S Leilei, A device for improving the safety of door opening [J]. Automob. Appl. Tech. 9, 274-275 (2016) (in Chinese)

12. H Kurtaran, M Buyuk, A Eskandarian, Ballistic impact simulation of GT model vehicle door using finite element method [J]. Theor. Appl. Fract. Mech. 40(2), 113-121 (2003)

13. TQ Fan, J Zhang, QJ Zhao, et al., Simulation modeling of vehicle front crash based on benchmark technology []. Bus Tech. Res. 32(3), 12-14 (2010) (in Chinese)

14. Hong Qiu-cai, Liu Wei-guo, Zhou Da-yong, et al. A Study of Restraint Simulation Based on LS-DYNA Application [C]. The 11th China CAE Annual Conference. 2015

15. D Bendjaballah, A Bouchoucha, ML Sahli, J-C Gelin, Numerical modelling and experimental analysis of the passenger side airbag deployment in out-of-position [J]. Int. J. Crashworthiness 22(5), 1-14 (2017)

16. Gu Chao-yang, Li Bi-hao. The Method of Folding Airbag by LS-DYNA and PRIMER [C]. The 11th China CAE Annual Conference. 2015.

\section{Submit your manuscript to a SpringerOpen ${ }^{\circ}$ journal and benefit from:}

- Convenient online submission

- Rigorous peer review

- Open access: articles freely available online

- High visibility within the field

- Retaining the copyright to your article

Submit your next manuscript at $\boldsymbol{\nabla}$ springeropen.com 